\title{
ON COLUMN-CONVEX AND CONVEX CARLITZ POLYOMINOES
}

\author{
TOUFIK MANSOUR, REZA RASTEGAR, AND ARMEND SH. SHABANI
}

\begin{abstract}
In this paper, we introduce and study Carlitz polyominoes. In particular, we show that, as $n$ grows to infinity, asymptotically the number of

(1) column-convex Carlitz polyominoes with perimeter $2 n$ is

$$
\frac{9 \sqrt{2}(14+3 \sqrt{3})}{2704 \sqrt{\pi n^{3}}} 4^{n} .
$$
\end{abstract}

(2) convex Carlitz polyominoes with perimeter $2 n$ is

$$
\frac{n+1}{10}\left(\frac{3+\sqrt{5}}{2}\right)^{n-2} \text {. }
$$

\section{INTRODUCTION AND PRELIMINARIES}

Take the upright square lattice and call each of its squares, together with its sides, a cell. A squared lattice polyomino, or simply polyomino, is a finite collection of edgeconnected cells in the lattice. The polyominoes were first introduced and studied by Golomb in 1953 [14 in certain mathematical recreational problems, and soon, they became central objects in the study of Ising model, Pott model, percolation theory, branched polymers [15, 24 26]), the mechanics of macromolecules [28], tiling problems [4, 5, 13, 21, 23], and enumeration problems [2, 7, 9]. We refer to [20,27] and references therein for a review of early literature.

The enumeration of polyominoes in the general case is an intractable and long-standing open problem. To tackle the problem, many authors have analyzed different subsets of polyominoes assuming additional constraints such as convexity, in which the enumeration has been conducted with respect to several statistics; including but not limited to the area, the perimeter, the outer/inner perimeter, and the corners. The techniques advised in enumeration of these subsets are diverse and range from bijective methods [2, 8, and algorithmic enumeration [7, 18, 19], to various forms of decompositions (see [16] and references wherein). See Table 1 for few examples.

Our objective in this paper is to introduce Carlitz polyominoes and enumerate them with some additional convexity constraints. To that goal, we first recall some standard definitions. A column (resp. row) of a polyomino is the collection of all cells in the polyomino belonging to an infinite vertical (resp. horizontal) array of cells in the lattice. For any polyomino $\nu$, let $h(\nu)$ and $v(\nu)$ to be the number of rows and columns of $\nu$, respectively. We define the perimeter of $\nu$ as $2(h(\nu)+v(\nu))$. A polyomino is said to be column-convex (resp. row convex) if each of its columns (resp. rows) is a single contiguous block of cells. We say a polyomino is convex if it is both column-convex and

2010 Mathematics Subject Classification. 05B50; 05A16.

Key words and phrases. Carlitz polyominoes; 


\begin{tabular}{c||l} 
Description & Ref. \\
\hline \hline Convex polyominoes & {$[9]$} \\
Column convex polyominoes & {$[10,11,[16]$} \\
$k$-convex polyominoes & {$[3]$} \\
Smooth polyominoes & {$[1]$} \\
Bargraphs & {$[1]$} \\
Directed animals on a strip & {$[15]$}
\end{tabular}

TABLE 1. Various classes of the polyominoes

row convex. We further assume that the cells are all squares of size one and equip the lattice with the Cartesian system, in which the bottom-left corner of the bottom cell of the leftmost column at the origin. Numerate the columns from the left side to the right side with the leftmost column counted as "1". Let $\nu$ be any nonempty polyomino with $m$ columns. We say that the bottom (resp. top) cell of the $i$ th column of $\nu$ is at the position $k$ if it lays on (resp. below) and touches the line $y=k$ and we denote this by $b_{i}(\nu)=k\left(\right.$ resp. $\left.u_{i}(\nu)=k\right)$, for $i=1,2, \ldots, m$. Define

$$
\begin{aligned}
& B(\nu)=\left|\left\{i \mid b_{i}(\nu)=b_{i+1}(\nu), i=1,2, \ldots, m-1\right\}\right|, \\
& U(\nu)=\left|\left\{i \mid u_{i}(\nu)=u_{i+1}(\nu), i=1,2, \ldots, m-1\right\}\right|,
\end{aligned}
$$

and refer to $B(\nu)$ and $U(\nu)$ as bottom levels and top levels, respectively.

A polyomino $\nu$ is said to be a Carlitz polyomino if $B(\nu)=U(\nu)=0$. A polyomino is said to be a convex (resp. column-convex) Carlitz polyomino if it is both convex (resp. column-convex) and Carlitz. See Figure 1 for a column-convex Carlitz polyominoe.

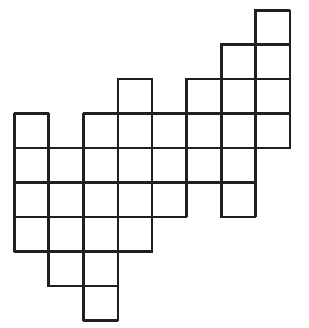

Figure 1. An example of column-convex Carlitz polyomino.

We remark that smooth polyominoes introduced in [17] are somewhat related to Carlitz polyominoes. For each smooth polyomino, the top and bottom positions of the columns cannot change by more than one unit going from each column to its neighbors. By contrast, the top and bottom positions in a Carlitz polyomino changes by at least one unit going from each column to its neighbors.

The paper is organized as follows. Section 2 is devoted to the enumeration of the column-convex Carlitz polyominoes. We first obtain an explicit formula for the generating function of the number of these polyominoes according to their perimeter. Then, Corollary 2.3 yields that the number of column-convex Carlitz polyominoes with the perimeter $2 n$ is asymptotic to

$$
\frac{9 \sqrt{2}(14+3 \sqrt{3})}{2704 \sqrt{\pi n^{3}}} 4^{n}
$$


as $n$ grows to infinity. In Section 3 , the convex Carlitz polyominoes are counted. We obtain the generating function for the number of convex Carlitz polyominoes according to their perimeters. Then, the explicit form is used to show that, as $n$ goes to infinity, the number of convex Carlitz polyominoes of the perimeter $2 n$ is asymptotic to

$$
\frac{n+1}{10}\left(\frac{3+\sqrt{5}}{2}\right)^{n-2}
$$

\section{Enumeration of COlumn-Convex Carlitz polyominoes}

In this section, we consider certain decompositions of the column-convex polyominoes and establish a functional system of linear combinations of multivariate series of forms $A\left(t_{1}, \ldots, t_{r} ; x_{1}, \ldots, x_{s}\right)$. These forms do not depend on $x_{1}, \ldots, x_{s}$, simultaneously and, their coefficients are referred to as kernels [29]. A systematic approach to solve these type of equations is given in [1]. We conduct all of our calculations and manipulations having the following facts in mind. Let $\mathbb{Q}, \mathbb{Q}\left[x_{1}, \ldots, x_{s}\right]$, and $\mathbb{Q}\left[\left[x_{1}, \ldots, x_{s}\right]\right]$ denote, respectively, the field of rational numbers, the ring of polynomials in $x_{1}, \ldots, x_{s}$, and the ring of formal power series in $x_{1}, \ldots, x_{s}$ with coefficients in $\mathbb{Q}$. Recall that a series $A \in \mathbb{Q}\left[x_{1}, \ldots, x_{s}\right]\left[\left[t_{1}, \ldots, t_{r}\right]\right]$ is $D$-finite if its partial derivatives span a finite dimensional vector space over the field of rational functions in $t_{1}, \ldots, t_{r}$ with coefficients in $\mathbb{Q}\left[x_{1}, \ldots, x_{s}\right]$. Note that any algebraic series is $D$-finite. The specializations of a $D$-finite series, obtained by assigning values in $\mathbb{Q}$ to a subset of variables, are $D$-finite, if well-defined. Moreover, if $A$ is $D$-finite, then any substitution $x_{i}=1$ with $i \in I \subseteq[s]$ or/and $x_{i}=x_{j}$ for $(i, j) \in I \times I \subseteq[s] \times[s]$ into $A$ is also $D$-finite. One last remark is that to derive asymptotic forms for our results, we apply singularity analysis (see [12, Section VI] for a comprehensive review). All calculations for asymptotic analysis require an appropriate domain of complex number $\mathbb{C}$. We omit the details for the sake of brevity as it is standard.

For the rest of this paper, we use $x, y, p$, and $q$ to mark $h(),. v(),. B($.$) and U($.$) ,$ respectively, while defining the corresponding generating functions. This section follows the methodology implemented in [6] closely. Define $\mathcal{C C P}$ to be the set of all nonempty column-convex polyominoes. Similarly, set $\mathcal{C C P}{ }_{a}$ to be the set of polyominoes in $\mathcal{C C P}$ with $a$ cells in their first columns. We define $F_{a}:=F_{a}(x, y, p, q)$ to be the generating function for the polyominoes in $\mathcal{C C P}_{a}$ according to $h(),. v(),. B($.$) , and U($.$) ; that is,$

$$
F_{a}(x, y, p, q):=\sum_{\nu \in \mathcal{C C P} \mathcal{P}_{a}} x^{h(\nu)} y^{v(\nu)} p^{B(\nu)} q^{U(\nu)}
$$

Next, decompose each $\nu \in \mathcal{C C P}_{a}$ by considering the size and the bottom position of its second column (if any), as described in Figure 2.

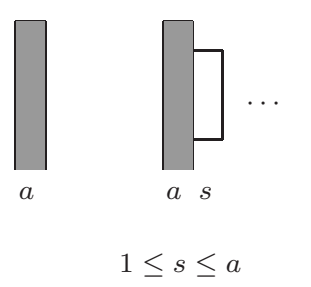

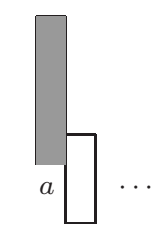

$s \geq 2$

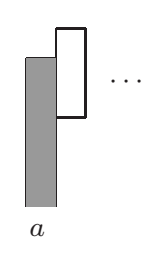

$s \geq 2$

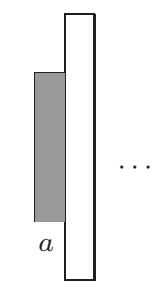

$s \geq a+2$

Figure 2. Decomposition of a polyomino in $\mathcal{C C P}_{a}$. 
By a careful analysis of these cases, for each $a \geq 1$, we may write

$$
\begin{aligned}
F_{a} & =x y^{a}+\sum_{s=1}^{a-1}(a-1-s+p+q) x y^{a-s} F_{s}+p q x F_{a} \\
& +2 \sum_{s=2}^{a} x \frac{y^{a+1-s}-y^{a}}{1-y} F_{s}+\sum_{s \geq a+1} x\left(2 \frac{y-y^{a}}{1-y}+p+q\right) F_{s}+\sum_{s \geq a+2}(s-1-a) x F_{s} .
\end{aligned}
$$

Now, define

$$
F(u)=F(u ; x, y, p, q)=\sum_{a \geq 1} F_{a} u^{a-1}
$$

By multiplying the last recurrence by $u^{a-1}$ and summing over all $a \geq 1$, we obtain the following result.

Lemma 2.1. The generating function $F(u)=F(u ; x, y, p, q)$ satisfies

$$
\begin{aligned}
& K(u ; x, y, p, q) F(u) \\
& =\frac{x y}{1-y u}+\frac{x}{1-u}\left(\frac{2 y u}{1-y u}+p+q-\frac{1}{1-u}\right) F(1)+\left.\frac{x}{1-u} \frac{\partial}{\partial u} F(u)\right|_{u=1},
\end{aligned}
$$

where $K(u ; x, y, p, q)=1-\frac{\left(q y u(u-1)-y u^{2}-q u+2 y u+q-1\right)\left(p y u(u-1)-y u^{2}-p u+2 y u+p-1\right) x}{(1-u)^{2}(1-y u)^{2}}$.

Note that by setting $q=p$ in Lemma 2.1, we get

$$
\begin{aligned}
& K(u ; x, y, q, q) F(u) \\
& =\frac{x y}{1-y u}+\frac{x}{1-u}\left(\frac{2 y u}{1-y u}+2 q-\frac{1}{1-u}\right) F(1)+\left.\frac{x}{1-u} \frac{\partial}{\partial u} F(u)\right|_{u=1},
\end{aligned}
$$

where

$$
K(u ; x, y, q, q)=1-\frac{\left(q y u(u-1)-y u^{2}-q u+2 y u+q-1\right)^{2} x}{(1-u)^{2}(1-y u)^{2}} .
$$

To solve this functional equation, we apply kernel method (see [1]). To that end, setting $K(u ; x, y, q, q)=0$ yields

$$
\mp 1+(q-1) \sqrt{x}+(( \pm 1-\sqrt{x} q)(y+1)+2 \sqrt{x} y) u+(\mp y+(q-1) \sqrt{x} y) u^{2}=0 .
$$

Solving for $u$, the roots of this equation are

$$
u_{ \pm}=\frac{1-\sqrt{1-\frac{4 y\left((q \sqrt{x} \pm 1)^{2}+\sqrt{x}(2 q-1)(\mp 2-\sqrt{x})\right)}{((1 \mp \sqrt{x} q)(y+1) \pm 2 \sqrt{x} y)^{2}}}}{\frac{2 y(1+\sqrt{x}(q-1))}{((1 \mp \sqrt{x} q)(y+1) \pm 2 \sqrt{x} y)^{2}}} .
$$

By substituting $u=u_{ \pm}$into Lemma 2.1, solving for $F(1 ; x, y, q, q)$, and evaluating $\left.\frac{\partial}{\partial u} F(u ; x, y, q, q)\right|_{u=1}$, we obtain the main result of this section.

Theorem 2.2. The generating function $F(1 ; x, y, q, q)$ is given by

$$
\begin{aligned}
& \frac{\left(u_{+}-1\right)\left(u_{-}-1\right) y(y-1)}{u_{+} u_{-} y(y-2)+\left(u_{+}+u_{-}\right) y-2 y+1} \\
& =x y+x y^{2}+q^{2} x^{2} y+x y^{3}+\left(q^{2}+4 q\right) x^{2} y^{2}+q^{4} x^{3} y+\cdots .
\end{aligned}
$$


In particular, $F(1 ; x, y, 0,0)$ counts the number of the column-convex Carlitz polyominoes according to $h($.$) and v($.$) , and is given by$

$$
\begin{aligned}
& \frac{\left(v_{+}-1\right)\left(v_{-}-1\right) y(y-1)}{v_{+} v_{-} y(y-2)+\left(v_{+}+v_{-}\right) y-2 y+1} \\
& =x y+x y^{2}+x y^{3}+x y^{4}+4 x^{2} y^{3}+x y^{5}+12 x^{2} y^{4}+x^{3} y^{3}+\cdots,
\end{aligned}
$$

where $v_{ \pm}=\left.u_{ \pm}\right|_{q=0}$.

Note that the generating function for the number of the column-convex Carlitz polyominoes according to the perimeter is simply given by

$$
\begin{aligned}
F\left(1 ; x^{2}, x^{2}, 0,0\right)= & \frac{\left(1-x^{2}\right)\left(20 x^{6}-45 x^{4}+42 x^{2}-21\right)}{4\left(8 x^{6}-27 x^{4}+36 x^{2}-18\right)} \\
& +\frac{(1-x)\left(2 x^{3}-9 x^{2}+9\right)(x+1)^{2}}{4\left(8 x^{6}-27 x^{4}+36 x^{2}-18\right)} \sqrt{4 x^{4}+x^{2}+1-2 x\left(1+2 x^{2}\right)} \\
& -\frac{(1+x)\left(2 x^{3}+9 x^{2}-9\right)(x-1)^{2}}{4\left(8 x^{6}-27 x^{4}+36 x^{2}-18\right)} \sqrt{4 x^{4}+x^{2}+1+2 x\left(1+2 x^{2}\right)} \\
& +\frac{3\left(x^{2}-1\right)^{2}}{4\left(8 x^{6}-27 x^{4}+36 x^{2}-18\right)} \sqrt{\left(4 x^{4}+x^{2}+1\right)^{2}-4 x^{2}\left(1+2 x^{2}\right)^{2}} .
\end{aligned}
$$

We end this section by extracting the coefficient of $x^{n}$ in $F(1 ; x, x, 0,0)$ and $\left.\frac{d}{d q} F(1 ; x, x, q, q)\right|_{q=1}$, for large $n$, by conducting singularity analysis of (2.1). The end result states that

Corollary 2.3. Asymptotically, as $n$ gets large,

(1) the number of column-convex Carlitz polyominoes with perimeter $2 n$ is $\frac{9 \sqrt{2}(14+3 \sqrt{3})}{2704 \sqrt{\pi n^{3}}} 4^{n}$.

(2) the total sum of $B+U$ over all column-convex polyominoes with perimeter $2 n$ is

$$
\frac{(1588-999 \sqrt{2}) \sqrt{5 \sqrt{2}-7}+6(51 \sqrt{2}-28) \sqrt{99 \sqrt{2}-140}}{2209 \sqrt{\pi n}}\left(\frac{3+2 \sqrt{2}}{2}\right)^{n} .
$$

\section{Enumeration of COnvex Carlitz polyominoes}

This section follows the approach developed in [16] closely. We define $\mathcal{C} \mathcal{P}^{t}$ to be the set of all nonempty convex polyominoes $\nu$, where for each column $j$ of $\nu, u_{s}(\nu) \leq u_{j}(\nu)$ for all $s \geq j+1$. Similarly, $\mathcal{C} \mathcal{P}^{b}$ is the set of all nonempty convex polyominoes $\nu$ such that for all columns $j$, and for all $s \geq j+1, b_{s}(\nu) \geq b_{j}(\nu)$. Set $\mathcal{C} \mathcal{P}^{b t}:=\mathcal{C} \mathcal{P}^{t} \cap \mathcal{C} \mathcal{P}^{b}$. By a clear upside down symmetry, there is a bijection between the set $\mathcal{C} \mathcal{P}^{t}$ and the set $\mathcal{C} \mathcal{P}^{b}$. See Figure 3, for an example of polyominoes in $\mathcal{C} \mathcal{P}^{t}$ and $\mathcal{C} \mathcal{P}^{b t}$. In addition, we let $\mathcal{C P}{ }_{a}^{t}, \mathcal{C P}{ }_{a}^{b}, \mathcal{C P}{ }_{a}^{b t}$, and $\mathcal{C P}{ }_{a}$ denote the set of polyominoes in $\mathcal{C} \mathcal{P}^{t}, \mathcal{C P}{ }^{b}, \mathcal{C P}{ }^{b t}$, and $\mathcal{C P}$ with $a$ cells in their first columns. Our enumeration is done as follows. The first step is to count the number of polyominoes in $\mathcal{C} \mathcal{P}^{b t}$ with respect to the statistics of interest $h(),. v(),. U($.$) , and B($.$) . Then, for the second step, we extend the result from$ $\mathcal{C} \mathcal{P}^{b t}$ to $\mathcal{C} \mathcal{P}^{t}$ and $\mathcal{C} \mathcal{P}^{b}$. This can be done since the corresponding generating functions can be written recursively in terms of the corresponding generating functions in $\mathcal{C} \mathcal{P}^{b t}$. When this is done, the last step is to obtain the results for $\mathcal{C P}$ by lifting up the result obtained for $\mathcal{C} \mathcal{P}^{t}$ and $\mathcal{C} \mathcal{P}^{b}$. 

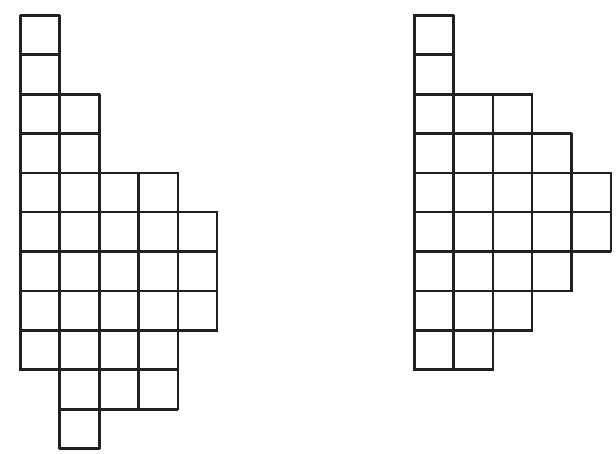

Figure 3. An example of polyominoes in (left) $\mathcal{C} \mathcal{P}^{t}$ (right) $\mathcal{C} \mathcal{P}^{b t}$

Let $G_{a}:=G_{a}(x, y, p, q)$ be the generating functions of $h(),. v(),. B($.$) , and U($.$) over$ $\mathcal{C P}$; that is,

$$
G_{a}:=G_{a}(x, y, p, q)=\sum_{\nu \in \mathcal{C} \mathcal{P}_{a}} x^{h(\nu)} y^{v(\nu)} p^{B(\nu)} q^{U(\nu)} .
$$

We define $G_{a}^{t}, G_{a}^{b}, G_{a}^{b t}$ similarly for the set of polyominoes $\mathcal{C P}{ }_{a}^{b}, \mathcal{C P} \mathcal{P}_{a}^{t}, \mathcal{C} \mathcal{P}_{a}^{b t}$, respectively. Moreover, we define the generating function $G(u)=G(u ; x, y, p, q)=\sum_{a \geq 1} G_{a} u^{a-1}$. Additionally, we define $G^{b}(u), G^{t}(u)$ and $G^{b t}(u)$.

Consider a polyomino $\nu$ in $\mathcal{C} \mathcal{P}_{a}^{b t}$ with the second column of size $s$ (if any). If second column does not exist then the contribution to $G_{a}^{b t}$ is exactly $x y^{a}$. If second column exists then $u_{2}(\nu) \leq u_{1}(\nu)$ and $b_{2}(\nu) \geq b_{1}(\nu)$, so there are exactly $a+1-s$ ways of gluing the second column to the first column. By considering all four cases

(1) $u_{2}(\nu)<u_{1}(\nu), b_{2}(\nu)>b_{1}(\nu)$

(2) $u_{2}(\nu)<u_{1}(\nu), b_{2}(\nu)=b_{1}(\nu)$,

(3) $u_{2}(\nu)=u_{1}(\nu), b_{2}(\nu)>b_{1}(\nu)$, and

(4) $u_{2}(\nu)=u_{1}(\nu), b_{2}(\nu)=b_{1}(\nu)$,

we obtain

$$
G_{a}^{b t}=x y^{a}+\sum_{s=1}^{a-1}(a-1-s+p+q) x y^{a-s} G_{s}^{b t}+p q x G_{a}^{b t} .
$$

By multiplying by $u^{a-1}$ and summing over $a \geq 1$, we obtain

$$
G^{b t}(u)=\frac{x y}{1-y u}+\frac{x y u(-y u(p+q-1)+p+q)}{(1-y u)^{2}} G^{b t}(u)+p q x G^{b t}(u) .
$$

Hence,

$$
G^{b t}(u ; x, y, p, q)=\frac{\frac{x y}{1-y u}}{1-p q x-\frac{x y u(-y u(p+q-1)+p+q)}{(1-y u)^{2}}} .
$$

Now, let us find a formula for $G^{t}(u)$. Any nonempty polyomino $\nu \in \mathcal{C P}_{a}^{t}$ can be decomposed as described in Figure 4. Therefore, the generating function $G_{a}^{t}(u)$ satisfies 


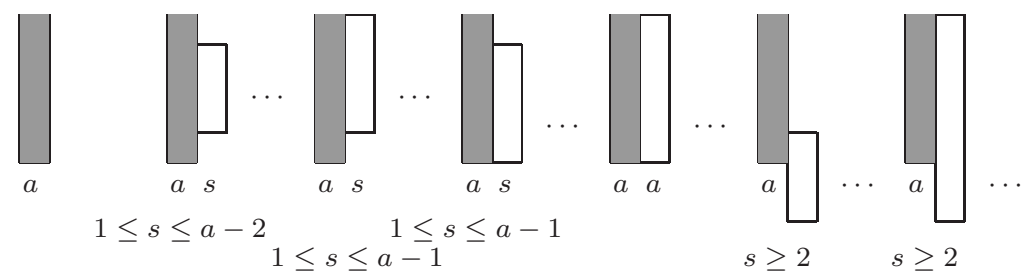

Figure 4. Decomposition of a polyomino in $\mathcal{C P}_{a}^{t}$.

$$
\begin{aligned}
G_{a}^{t} & =x y^{a}+\sum_{s=1}^{a-1}(a-1-s) x y^{a-s} G_{s}^{b t}+q \sum_{s=1}^{a-1} x y^{a-s} G_{s}^{b t}+p \sum_{s=1}^{a-1} x y^{a-s} G_{s}^{t} \\
& +p q x G_{a}^{t}+\sum_{s=2}^{a} x \frac{y^{a+1-s}-y^{a}}{1-y} G_{s}^{t}+\sum_{s \geq a+1} x\left(\frac{y-y^{a}}{1-y}+q\right) G_{s}^{t} .
\end{aligned}
$$

By multiplying this recurrence by $u^{a-1}$ and summing over $a \geq 1$, we obtain the equation

$$
\begin{aligned}
& \left(1-p q x+\frac{q x}{1-u}-\frac{p x y u}{1-y u}+\frac{x y u}{(1-u)(1-y u)}\right) G^{t}(u) \\
& =\frac{x y}{1-y u}+\left(\frac{x y^{2} u^{2}}{(1-y u)^{2}}+\frac{q x y u}{1-y u}\right) G^{b t}(u)+\frac{x y u+q x(1-y u)}{(1-u)(1-y u)} G^{t}(1),
\end{aligned}
$$

which will be solved by using the kernel method (see the beginning of Section 2). First, by finding the roots of the equation

$$
1-p q x+\frac{q x}{1-u^{\prime}}-\frac{p x y u^{\prime}}{1-y u^{\prime}}+\frac{x y u^{\prime}}{\left(1-u^{\prime}\right)\left(1-y u^{\prime}\right)}=0
$$

for variable $u$, we get

$$
u^{\prime}=\frac{1-\sqrt{1-\frac{4 y(1+p(1-q) x)(1+q(1-p) x)}{(1+y-p q x-x y(1-p)(1-q))^{2}}}}{\frac{2 y(1+p(1-q) x)}{1+y-p q x-x y(1-p)(1-q)}} .
$$

Then, setting $u=u^{\prime}$ in last functional equation yields

$$
G^{t}(1)=\frac{y\left(u^{\prime}-1\right)}{y u^{\prime}+q\left(1-y u^{\prime}\right)}+\frac{y u^{\prime}\left(u^{\prime}-1\right)}{1-y u^{\prime}} G^{b t}\left(u^{\prime}\right) .
$$

Thus, by (3.1), we can state the following result.

Lemma 3.1. The generating function $G^{t}(u ; x, y, p, q)$ is given by (3.2), where the generating function $G^{t}(1 ; x, y, p, q)$ is given by

$$
G^{t}(1)=\frac{y\left(u^{\prime}-1\right)}{y u^{\prime}+q\left(1-y u^{\prime}\right)}+\frac{x y^{2} u^{\prime}\left(u^{\prime}-1\right)}{(1-p q x)\left(1-y u^{\prime}\right)^{2}-x y u^{\prime}(p+q)\left(1-y u^{\prime}\right)-x y^{2} u^{\prime 2}} .
$$

Next, we find the generating function $G(1 ; x, y, p, q)$ based on $G^{t}$ and $G^{b}$. To that goal, we decompose each convex polyomino as described in Figure 5. Considering these 

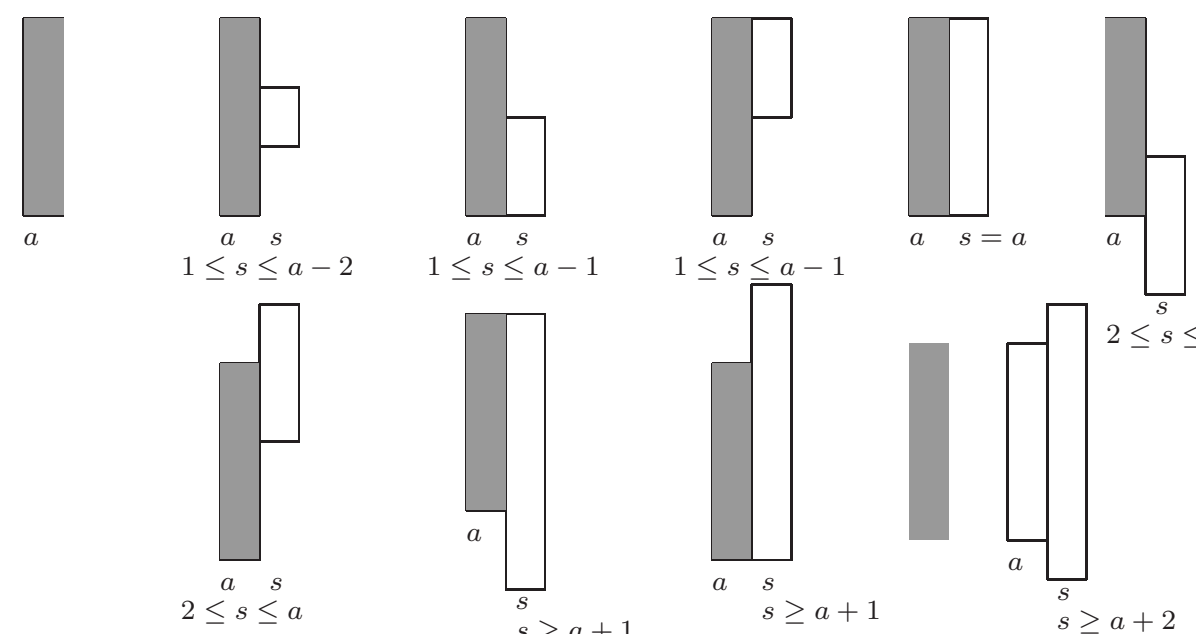

FiguRE 5. Decomposition of a polyomino in $\mathcal{C P}_{a}$

cases, we can rewrite $G_{a}(x, y, t)$ as

$$
\begin{aligned}
G_{a} & =x y^{a}+\sum_{s=1}^{a-1}(a-1-s) x y^{a-s} G_{b}^{b t}+p \sum_{s=1}^{a-1} x y^{a-s} G_{b}^{t}+q \sum_{s=1}^{a-1} x y^{a-s} G_{s}^{b} \\
& +x G_{a}+\sum_{s=2}^{a} x\left(y^{a-1}+\cdots+y^{a+1-s}\right) G_{s}^{t}+\sum_{s=2}^{a} x\left(y^{a-1}+\cdots+y^{a+1-s}\right) G_{s}^{b} \\
& +\sum_{s \geq a+1} x\left(y^{a-1}+\cdots+y\right) G_{s}^{t}+\sum_{s \geq a+1} x\left(y^{a-1}+\cdots+y\right) G_{s}^{b}+(p+q) \sum_{s \geq a+1} x G_{s} \\
& +\sum_{s \geq a+1}(s-1-a) x G_{s} .
\end{aligned}
$$

By multiplying by $u^{a-1}$ and summing over $a \geq 1$, we obtain

$$
\begin{aligned}
& \left(1-p q x+\frac{(p+q) x}{1-u}-\frac{x}{(1-u)^{2}}\right) G(u) \\
= & \frac{x y}{1-y u}+\frac{x y^{2} u^{2}}{(1-y u)^{2}} G^{b t}(u)+\frac{x y u}{1-y u}\left(p G^{t}(u)+q G^{b}(u)\right) \\
+ & \frac{x y u}{(1-u)(1-y u)}\left(G^{t}(1)+G^{b}(1)-G^{t}(u)-G^{b}(u)\right) \\
+ & \frac{(p+q) x}{1-u} G(1)-\frac{x}{(1-u)^{2}} G(1)+\left.\frac{x}{1-u} \frac{\partial}{\partial u} G(u)\right|_{u=1} .
\end{aligned}
$$

Recall $G^{b}(u ; x, y, p, q)=G^{t}(u ; x, y, q, p)$ by a symmetry argument. Also, note that the roots of the kernel

$$
K(u)=1-p q x+\frac{(p+q) x}{1-u}-\frac{x}{(1-u)^{2}}
$$

are

$$
u_{ \pm}=1+\frac{(p+q) x \pm \sqrt{(p-q)^{2} x^{2}+4 x}}{2(1-p q x)} .
$$

By inserting $u=u_{+}$and $u=u_{-}$, one at the time, into the functional equation (3.4), we obtain a system of two equations with variables $G(1)$ and $\left.\frac{\partial}{\partial u} G(u)\right|_{u=1}$. Solving this system yields 
Theorem 3.2. The generating function $G(1 ; x, y, p, q)$ is given by

$$
\begin{aligned}
& \frac{y(y-1)\left(1-u_{+}\right)\left(1-u_{-}\right)}{\left(1-y u_{+}\right)\left(1-y u_{-}\right)} \\
& +\frac{y^{2}\left(1-u_{+}\right)\left(1-u_{-}\right)}{u_{+}-u_{-}}\left(\frac{u_{+}^{2}\left(1-u_{+}\right) G^{b t}\left(u_{+} ; x, y, p, q\right)}{\left(1-y u_{+}\right)^{2}}-\frac{u_{-}^{2}\left(1-u_{-}\right) G^{b t}\left(u_{-} ; x, y, p, q\right)}{\left(1-y u_{-}\right)^{2}}\right) \\
& -\frac{y u_{+}\left(1-u_{+}\right)\left(1-u_{-}\right)}{\left(u_{+}-u_{-}\right)\left(1-y u_{+}\right)}\left(\left(1-p\left(1-u_{+}\right)\right) G^{t}\left(u_{+} ; x, y, p, q\right)+\left(1-q\left(1-u_{+}\right)\right) G^{t}\left(u_{+} ; x, y, q, p\right)\right) \\
& +\frac{y u_{-}\left(1-u_{+}\right)\left(1-u_{-}\right)}{\left(u_{+}-u_{-}\right)\left(1-y u_{-}\right)}\left(\left(1-p\left(1-u_{-}\right)\right) G^{t}\left(u_{-} ; x, y, p, q\right)+\left(1-q\left(1-u_{-}\right)\right) G^{t}\left(u_{-} ; x, y, q, p\right)\right) \\
& +\frac{y\left(1-u_{+}\right)\left(1-u_{-}\right)}{\left(1-y u_{+}\right)\left(1-y u_{-}\right)}\left(G^{t}(1 ; x, y, p, q)+G^{b}(1 ; x, y, q, p)\right),
\end{aligned}
$$

where $G^{b t}(u ; x, y, p, q), G^{t}(u ; x, y, p, q)$ and $G^{t}(1 ; x, y, p, q)$ are given by (3.1), (3.2) and (3.3), respectively.

As a corollary, the generating function $G(1 ; x, x, q, q)$ is given by

$$
\begin{aligned}
& \frac{x^{2} A}{\left(q^{2} x^{2}-q^{2} x-2 q x^{2}+x^{2}-3 x+1\right)^{2}\left(q^{2} x^{2}-q^{2} x-2 q x^{2}+x^{2}+x+1\right)^{2}} \\
& -\frac{x^{4}\left(q^{3} x^{2}-q^{3} x-3 q^{2} x^{2}+q^{2} x+3 q x^{2}-q x-x^{2}+q+x+1\right)^{2}}{\left(q^{2} x^{2}-q^{2} x-2 q x^{2}+x^{2}-3 x+1\right)^{3 / 2}\left(q^{2} x^{2}-q^{2} x-2 q x^{2}+x^{2}+x+1\right)^{3 / 2}} \\
& =x^{2}+\left(q^{2}+1\right) x^{3}+\left(q^{4}+q^{2}+4 * q+1\right) x^{4}+\left(q^{2}+1\right)\left(q^{4}+8 q+5\right) x^{5}+\cdots,
\end{aligned}
$$

where

$$
\begin{aligned}
A & =1-3\left(q^{2}+1\right) x+q\left(3 q^{3}+10 q-2\right) x^{2}-\left(q^{2}+1\right)\left(q^{4}+11 q^{2}-6 q-4\right) x^{3} \\
& +(q-1)\left(6 q^{5}+17 q^{3}-7 q^{2}-5 q-3\right) x^{4}-\left(q^{2}+1\right)\left(q^{4}+11 q^{2}-6 q-4\right)(q-1)^{2} x^{5} \\
& +q\left(3 q^{3}+10 q-2\right)(q-1)^{4} x^{6}-3\left(q^{2}+1\right)(q-1)^{6} x^{7}+(q-1)^{8} x^{8} .
\end{aligned}
$$

By considering $G(1 ; x, x, 0,0)$, we show that the generating function for the number of convex Carlitz polyominoes according to their perimeters is given by

$$
\frac{x^{4}\left(x^{16}-3 x^{14}+4 x^{10}+3 x^{8}+4 x^{6}-3 x^{2}+1\right)}{\left(x^{4}-3 x^{2}+1\right)^{2}\left(x^{4}+x^{2}+1\right)^{2}}-\frac{x^{8}\left(x^{4}-x^{2}-1\right)^{2}}{\left(x^{4}-3 x^{2}+1\right)^{3 / 2}\left(x^{4}+x^{2}+1\right)^{3 / 2}} .
$$

Next, by singularity analysis of $G(1 ; x, x, 0,0)$, we get

Corollary 3.3. As $n$ grows to infinity, the number of convex Carlitz polyominoes with the perimeter $2 n$ is asymptotic to $\frac{n+1}{10}\left(\frac{3+\sqrt{5}}{2}\right)^{n-2}$.

Note that by Theorem 3.2 ,

$$
\left.\frac{d}{d q} G(1 ; x, x, q, q)\right|_{q=1}=\frac{2 x^{3}\left(16 x^{4}-12 x^{3}+10 x^{2}-5 x+1\right)}{(1-4 x)^{3}}+\frac{4 x^{4}\left(4 x^{2}-1\right)}{(1-4 x)^{5 / 2}} .
$$

We conclude this section by stating that singularity analysis of $\left.\frac{d}{d q} G(1 ; x, x, q, q)\right|_{q=1}$ also yields

Corollary 3.4. The total sum of $B+U$ over all convex polyominoes with perimeter $2 n$ is asymptotic to $n^{2} 4^{n-4}$. 


\section{REFERENCES}

[1] C. Banderier, M. Bousquet-Mélou, A. Denise, P. Flajolet, D. Gardy and D. Gouyou-Beauchamps, Generating functions for generating trees, Disc. Math., 246:1-3 (2000), 29-55.

[2] E. Barcucci, A. Frosini and S. Rinaldi, Direct-convex polyominoes: ECO method and bijective results, Proc. of Formal Power Series and Algebraic Combinatorics, Melbourne, 2002.

[3] A. Boussicault, S. Rinaldi, S. Socci, The number of directed $k$-convex polyominoes, Disc. Math., 343:3 (2020), \#111731.

[4] D. Beauquier, M. Nivat, É. Remila, M. Robson, Tiling figures of the plane with two bars, Comput. Geometry. Theory and Appl., 5:1 (1995), 1-25.

[5] R. Berger, The undecidability of the domino problem, Memoirs Amer. Math. Soc., 66 (1966), 72.

[6] N. Cakić, T. Mansour, G. Yıldırım, A decomposition of column-convex polyominoes and two vertex statistics, preprint.

[7] A. Conway, Enumerating $2 D$ percolation series by the finite-lattice method: theory, J. Phys. A, 28:2 (1995), 335-349.

[8] A. Del Lungo, M. Mirolli, R. Pinzani and S. Rinaldi, A bijection for directed-convex polyominoes, Proc. of DM-CCG 2001, Disc. Math. Theoret. Comput. Sci. AA, 2001, 133-144.

[9] M. Delest, X. G. Viennot, Algebraic languages and polyominoes enumeration, Theoret. Comput. Sci., 34 (1984), 169-206.

[10] S. Feretić, D. Svrtan, On the number of column-convex polyominoes with given perimeter and number of columns, 5th FPSAC Proc., Firenze (1993), 201-214.

[11] S. Feretić, A perimeter enumeration of column-convex polyominoes, Disc. Math. Theoret. Comput. Sci., 9 (2007), 57-84.

[12] P. Flajolet, R. Sedgewick, Analytic Combinatorics, Cambridge University Press, 2009.

[13] B. Grünbaum, G.C. Shephard, Tilings and patterns, W.H. Freeman and Company, New York, 1989.

[14] S. W. Golomb, Checker boards and polyominoes, Amer. Math. Monthly, 61 (1954), 675-682.

[15] V. Hakim, J.P. Nadal, Exact results for $2 D$ directed animals on a strip of finite width, J. Phys. A. Mathematical and General, 16:7 (1983), 213-218.

[16] T. Mansour, R. Rastegar, Convex polyominoes revisited: enumeration of outer site perimeter, interior vertices, and boundary vertices of certain degrees, J. of Diff. Eq. and App., 26:7 (2021), $1013-1041$.

[17] T. Mansour, A. Sh. Shabani, Smooth Column Convex Polyominoes, Submitted.

[18] I. Jensen, Enumerations of Lattice Animals and Trees, J. Stat. Phys., 102:3-4 (2001), 865-881.

[19] I. Jensen, A. J. Guttmann, Statistics of lattice animals (polyominoes) and polygons, J. Phys. A, 33:29 (2000), 257-263.

[20] A.J. Guttmann, Polygons, Polyominoes and Polycubes, Springer, Netherlands, 2009.

[21] D.A. Klarner, My life among the polyominoes, Nieuw Archief voor Wiskunde. Derde Serie, 29:2 (1981), 156-177.

[22] D.A. Klarner, Some results concerning polyominoes, The Fib. Quarterly, 3 (1965), 9-20.

[23] D.A. Klarner, Packing a rectangle with congruent n-ominoes, J. Combin. Theory, 7 (1969), 107115.

[24] V. Privman, N.M. Svrakic, Difference equations in statistical mechanics. I. Cluster statistics models, J. Stat. Phys., 51:5-6 (1988), 1091-1110.

[25] V. Privman, N.M. Svrakic, Directed models of polymers, interfaces, and clusters: scaling and finite-size properties, Springer-Verlag, Berlin, 1989.

[26] G. Viennot, Problémes combinatoires posés par la physique statistique, Astérisque, 121-122 (1985), 225-246.

[27] X.G. Viennot, A survey of polyominoes enumeration, 4th FPSAC Proc., Publications du LACIM, Institut Mittag-Leffler, 11 (1992), 399-420.

[28] H.N.V. Temperley, Combinatorial problems suggested by the statistical mechanics of domains and of rubber-like molecules, Phys. Rev., 103 (1956), 1-16.

[29] D. Zeilberger, The umbral transfer-matrix method: I. Foundations, J. Comb. Theory, Ser. A, 91 (2000), 451-463. 
Department of Mathematics, University of Haifa, 3498838 Haifa, Israel

Email address: tmansour@univ.haifa.ac.il

Occidental Petroleum Corporation, Houston, TX 77046 and Departments of Mathematics and Engineering, University of Tulsa, OK 74104, USA - Adjunct Professor

Email address: reza_rastegar2@oxy.com

Department of Mathematics, University of Prishtina, 10000 Prishtinë, Republic of Kosovo

Email address: armend.shabani@uni-pr.edu 